\title{
Atypical ductal hyperplasia is a multipotent precursor of breast carcinoma
}

Tanjina Kader ${ }^{1,2}$, Prue Hill ${ }^{3}$, Magnus Zethoven ${ }^{1}$, David L. Goode ${ }^{1,2}$, Kenneth Elder ${ }^{4}$, Niko Thio $^{1}$, Maria Doyle ${ }^{1}$, Timothy Semple ${ }^{1}$, Wajiha Sufyan $^{5}$, David J. Byrne ${ }^{1}$, Jia-Min B. Pang ${ }^{1}$, Anand Murugasu ${ }^{4}$, Islam M Miligy ${ }^{6}$, Andrew R Green ${ }^{6}$, Emad A Rakha ${ }^{6}$, Stephen B. Fox ${ }^{1}$, G. Bruce Mann ${ }^{4}$, Ian G. Campbell ${ }^{1,2,7 \dagger}$, Kylie L. Gorringe $e^{1,2,7 * \dagger}$

${ }^{1}$ Peter MacCallum Cancer Centre, 305 Grattan St, Melbourne, Australia, 3000. ${ }^{2}$ The Sir Peter MacCallum Department of Oncology, The University of Melbourne, Parkville, Australia, 3010. ${ }^{3}$ Department of Anatomical Pathology, St Vincent's Hospital, Fitzroy, Australia. ${ }^{4}$ The Breast Service, The Royal Women's Hospital, Melbourne, Australia. ${ }^{5}$ Territory Pathology, Royal Darwin Hospital, Darwin, NT. ${ }^{6}$ Nottingham Breast Cancer Research Centre, Division of Cancer and Stem Cells, School of Medicine, University of Nottingham and Department of Histopathology, Nottingham University Hospitals NHS Trust, City Hospital, Nottingham, UK. ${ }^{7}$ Department of Clinical Pathology, The University of Melbourne, Parkville, Australia, 3010.

$\dagger$ These authors contributed equally to this work

Running Title: Multipotent nature of atypical ductal hyperplasia

The authors declare no potential conflicts of interest

To whom correspondence should be addressed:

This is the author manuscript accepted for publication and has undergone full peer review but has not been through the copyediting, typesetting, pagination and proofreading process, which may lead to differences between this version and the Version of Record. Please cite this article as doi: $10.1002 /$ path.5262

This article is protected by copyright. All rights reserved. 
Dr. Kylie Gorringe; Research Division, Peter MacCallum Cancer Centre, 305 Grattan St, Melbourne, Australia, 3000; Phone: +613 855 96521;

Email: kylie.gorringe@petermac.org

\begin{abstract}
The current model for breast cancer progression proposes independent "low-grade (LG) like" and "high-grade (HG) like" pathways but lacks a known precursor to HG cancer. We applied low coverage whole genome sequencing to atypical ductal hyperplasia (ADH) with and without carcinoma to shed light on breast cancer progression. 14/20 isolated ADH cases harboured at least one copy number alteration (CNA), but had fewer aberrations than LG or HG ductal carcinoma in situ (DCIS). ADH carried more HG-like CNA than LG DCIS (eg. 8q gain). Correspondingly, 64\% (7/11) of ADH cases with synchronous HG carcinoma were clonally related, similar to LG carcinoma $(67 \%, 6 / 9)$. This study represents a significant shift in our understanding of breast cancer progression, with $\mathrm{ADH}$ as a common precursor lesion to the independent "low-grade like" and "high-grade like" pathways. These data suggest that ADH can be a precursor of HG breast cancer and that LG and HG carcinomas can evolve from a similar ancestor lesion. We propose that although LG DCIS may be committed to a LG molecular pathway, ADH may remain multipotent, progressing to either LG or HG carcinoma. This multipotent nature suggests that some ADH could be more clinically significant than LG DCIS, requiring biomarkers for personalising management.
\end{abstract}

This article is protected by copyright. All rights reserved. 
Keywords: atypical ductal hyperplasia, copy number, breast cancer progression, clonal, ductal carcinoma in situ, whole genome sequencing, pre-malignant breast lesions, benign breast disease

This article is protected by copyright. All rights reserved. 


\section{Introduction}

The current model for breast cancer progression proposes independent "low-grade like" and "high-grade like" pathways [1] where low-grade (LG) and high-grade (HG) invasive ductal carcinoma (IDC) progress from LG and HG ductal carcinoma in situ (DCIS), respectively $[1,2]$. Atypical ductal hyperplasia (ADH) is a common lesion associated with up to a $30 \%$ life-time risk of metachronous breast cancer [3,4]. ADH occurs within terminal ductal lobular units (TDLU) and appears as a proliferation of evenly spaced monomorphic epithelial cells that have well defined cell borders and generally rounded nuclei. The cells form "punched out" (cribriform-like) secondary lumens and/or micropapillae $[5,6]$. It is diagnostically challenging, lacking reproducibility across pathologists. ADH has been considered to be a general risk indicator, but a precursor for the LG pathway only, and thus, also of ER+ carcinoma only $[1,2]$. However, the genomic data was at odds with clinical observations that two-thirds of the cancers arising after a diagnosis of $\mathrm{ADH}$ are $\mathrm{HG}$ or intermediate grade (IG) [7,8]. Whether HG, HER2+ or ER- carcinoma could also develop from this lesion remains unclear.

While some studies have identified a few common genomic features of $\mathrm{ADH}$, a clear understanding of the molecular changes associated with ADH development has been limited by inadequately powered studies and/or low-resolution methodology [9-16].

Copy number alteration (CNA) is the dominant somatic genetic event in breast cancer $[1,17]$, and we previously used CNA to show that most DCIS is clonally related to co-existing IDC, validating DCIS as a direct precursor of IDC [18-20]. We hypothesised that a similar 
analysis of $\mathrm{ADH}$ would provide an insight into early clonal expansions and clarify whether it can be a precursor of HG breast cancer. We carried out CNA analysis using low-coverage whole genome sequencing (LCWGS) of cases of $\mathrm{ADH}$ without carcinoma (pure ADH) and ADH with co-existing carcinoma of different grades. Because of the difficulties in diagnosing $\mathrm{ADH}$, we consistently applied a strict histopathological definition in selecting the cohort.

\section{Methods}

\section{Tumour samples}

All cases of ADH without carcinoma (pure ADH) and ADH co-existing with carcinoma (synchronous $\mathrm{ADH}$ ) were identified from the Royal Melbourne Hospital (RMH) database from 1995 - 2015 with follow up until 2017 through the Victorian Cancer Registry. Cases were considered pure ADH when no carcinoma was reported in the 2-22 years preceding diagnosis (supplementary material, Table S1) as well as no carcinoma diagnosed within a year after ADH diagnosis. ADH from RMH were diagnosed by multiple pathologists with different levels of expertise, therefore, diagnostic haematoxylin and eosin (H\&E) stained tissue sections of $\mathrm{ADH}(\mathrm{n}=100)$ and synchronous $\mathrm{ADH}(\mathrm{n}=40)$ were reviewed by a specialist breast anatomical pathologist $\mathrm{PH}$. The criteria for $\mathrm{ADH}$ was according to Page et al. [6] and Lakhani et al. [5]: ADH is characterised by a proliferation within TDLU of a monomorphic population of epithelial cells with generally rounded nuclei that are evenly spaced and have well defined cell borders. The cells form "punched out" (cribriform-like) secondary lumens and/or micropapillae. The cells may grow in arcades or rigid bridges of uniform thickness (supplementary material, Figure S1). In distinguishing ADH from LG DCIS, the latter 
required complete involvement of $>2$ ducts or partial involvement of ducts $>2 \mathrm{~mm}$ in extent. Exclusion criteria were absence of atypical cells (i.e. usual ductal hyperplasia or UDH) (Figure S1), columnar cell change with UDH, and other early neoplasia with atypia such as flat epithelial atypia (FEA), radial scar or apocrine hyperplasia. Upon review, 40/100 pure ADH did not meet these criteria and were excluded, while 20/100 were considered LG DCIS and also excluded. Details of patient selection are shown in supplementary material, Figure $\mathrm{S} 2$.

$\mathrm{ADH}$ with synchronous carcinoma was confirmed on review in 30/40 cases. Cases were excluded when the "ADH" was deemed to be a part of the associated carcinoma (supplementary material, Figure S3). We placed any cases with features of IG carcinomas into the LG group if LG carcinoma was also present (e.g. LG DCIS with G2 IDC) and IG cases with HG carcinoma into the HG group. Two cases with solely IG carcinoma were arbitrarily allocated to the HG group prior to data analysis (supplementary material, Table S2 and Supplementary materials and methods).

Genomic data for LG DCIS cases $(n=10)$ and HG DCIS cases $(n=38)$ were derived from our previously published work [20,21], with new data from an additional 11 LG DCIS cases from the RMH, Peter MacCallum Cancer Centre and Nottingham University Hospitals NHS Trust, UK.

This study was conducted under ethical approval from the Peter MacCallum Cancer Centre (HREC \#12-64), Melbourne Health (HREC\# 2012.119) and the North West-Greater Manchester Central Research Ethics Committee 15/NW/0685. 


\section{Tissue microdissection and DNA extraction}

Neoplastic epithelial cells were micro-dissected from 8-10 micron H\&E stained sections (1520 per case) using the Roche Automated Tissue Dissection System to achieve $>50 \%$ tumour purity. A new reference H\&E slide was evaluated by pathologist JMP before dissection. Micro-dissected tissue was placed in buffer ATL and sonicated using the Covaris LE220 system, followed by proteinase K digest. DNA was extracted using the MagAttract ${ }^{\circledR}$ HMW DNA miniKit (Qiagen, Hilden, Germany) as described [22] except for four LG DCIS cases that were extracted using the All Prep Kit (Qiagen).

\section{NEBNext ${ }^{\circledR}$ Ultra $^{T M}$ II DNA library preparation and low coverage whole-genome sequencing (LCWGS)}

Low DNA input library preparation was performed with the NEBNext ${ }^{\circledR}$ Ultra ${ }^{\mathrm{TM}}$ II DNA Library Prep Kit (New England BioLabs ${ }^{\circledR}$ Inc., Ipswich, MA, USA) and used for LCWGS as previously [22]. An Illumina Nextseq500 (paired-end 75 bp reads) was used to run the pooled, normalized, indexed libraries according to the Illumina protocol. Sequencing depth ranged from $0.76-3.34 x$. (supplementary material, Table S3).

\section{Data analysis}

This article is protected by copyright. All rights reserved. 
Reads were aligned with bwa mem (v0.7.12-r1039) to hg19 after removal of sequencing primers by cutadapt (v1.7.1) as previously [22]. ControlFREEC (v6.7) [23] was used to estimate $\mathrm{CN}$ using $50 \mathrm{~kb}$ windows as described previously [22].

Data were imported into Nexus (v8, BioDiscovery Inc., Hawthorne, CA, USA) and segmented using SNP-FASST. CNA were called if the $\log _{2}$ ratio was \pm 0.15 . Blacklisted regions identified by Scheinin et al. [24] were filtered out as described previously [22]. FGA was calculated as described in supplementary material, Supplementary materials and methods..

CN segments were imported into Partek Genome Suite (Partek Inc., St. Louis, MO, USA) to perform unsupervised hierarchical clustering using Pearson dissimilarity and average linkage.

Lesions were classified as clonally related when sharing e 2 CNA with visually inspected shared breakpoints (supplementary material, Figures S4-S7) at $50 \mathrm{~kb}$ resolution, except one case where $\mathrm{ADH}$ and LG DCIS shared one rare CNA, gain of whole chromosome 8 . The “Clonality" package (R v3.5.1) [25-28] was also used to test clonality. Averaging of data was carried out to reduce the data resolution to 15,000 markers as recommended [25].

\section{Statistical analysis}

GraphPad Prism v7 (GraphPad, Inc., San Diego, CA, USA) was used to generate graphs and perform statistical tests as indicated. A $p$ value of $<0.05$ was considered significant.

\section{Results}

This article is protected by copyright. All rights reserved. 


\section{Pure ADH show genetic characteristics of both low and high grade carcinoma}

Pure ADH diagnoses $(n=20)$ were re-confirmed by extensive pathology review by $\mathrm{PH}$, including of the subsequent excision for those $\mathrm{ADH}$ cases diagnosed on core biopsies to reconfirm those lesions were not part of any LG DCIS (supplementary material, Table S1). All $\mathrm{ADH}$ were ER+ and CK5/6- (supplementary material, Figure S1). The median follow-up time was 6 years (2-22 years) with no patients recording a subsequent carcinoma in that period. $\mathrm{CN}$ profiling showed that $70 \%$ of pure $\mathrm{ADH}$ had at least one CNA with a median number of three events and a median fraction of the genome altered (FGA) of 5\% (0-19\%)

(Figure 1). The most prevalent $\mathrm{CN}$ changes were $16 \mathrm{q}$ loss $(65 \%)$, followed by $16 \mathrm{p}$ gain (35\%), 1q gain (25\%) and 8q gain (20\%) (Table 1).

We compared the spectrum and frequency of CNA in pure ADH with data from LG DCIS $(\mathrm{n}=21)$, HG DCIS $(\mathrm{n}=38)[20,21]$ and LG (grade 1) and HG (grade 3) IDC (METABRIC) [29]. LG and HG carcinoma (both DCIS and IDC) differ markedly in their CN profiles with $81 \%$ of LG carcinomas harbouring 16q loss compared with 32\% for HG cases (Figure 1D, Table 1). In pure $\mathrm{ADH}$, the $\mathrm{CN}$ profiles resemble those of $\mathrm{LG}$ breast cancers with respect to 16q loss (13/20 cases, 65\%) (Table 1), but were less likely to have concomitant 1q gain (4/20) than LG DCIS (10/21 with 1q+/16q-), more resembling HG DCIS (9/38).

Furthermore, some pure ADH cases showed a HG-like CN profile, with 8q gain (20\%) or 20q gain $(10 \%)$ (Table 1, Figure 1D). Partial or whole arm gain of $8 \mathrm{q}$ was more frequently observed in HG carcinoma (58\% HG DCIS, 53\% G3 IDC) compared with LG carcinoma cases (0\% LG DCIS, 15\% G1 IDC) (LG versus HG DCIS: $p<0.0001$, Fisher Exact test). There was a statistically significant difference observed between ADH and LG DCIS for 8q 
gain (ADH 4/20 case, LG DCIS 0/21; p=0.04, Fisher's Exact test). The HG-like CN profile of $\mathrm{ADH}$ was not limited to $8 \mathrm{q}$ gain, indeed $7 / 20 \mathrm{ADH}$ cases carried $\mathrm{CN}$ events statistically significantly enriched in HG versus LG DCIS, such as 20q gain, 17p loss or 18q loss (Figure 1A). Collectively, from these observations we hypothesised that ADH has the potential to progress directly to either LG or HG cancer.

\section{ADH is clonal to both low and high grade carcinoma}

We then investigated the clonal relationship of ADH co-existing with breast carcinoma of different grades. These ADH were defined based on their morphological appearance and cases were excluded if ADH was thought to be part of the carcinoma (supplementary material, Figure S3D, Table S1). We generated $\mathrm{CN}$ profiles for 20 such $\mathrm{ADH}$ cases and the synchronous ipsilateral carcinoma components (supplementary material, Table S1). Overall, $65 \%$ of $\mathrm{ADH}$ was found to be clonal with the synchronous DCIS and/or IDC. The rate of clonality was not significantly different for synchronous LG (6/9 cases) and synchronous HG (7/11 cases) carcinoma ( $>0.99$, Figure 2A). This result was not substantially different if cases with IG carcinoma were considered separately (supplementary material, Table S2): clonality rates were $4 / 6$ for purely LG carcinomas, $6 / 8$ for carcinomas with any IG/G2 component, and 3/6 for purely HG carcinomas. There was no difference observed for the size of the synchronous carcinoma (mean size clonal $=14.9 \mathrm{~mm}$, non-clonal $=18.7 \mathrm{~mm}, \mathrm{p}=0.93)$ or whether the $\mathrm{ADH}$ was located in the same or different tissue block ( $\mathrm{p}=0.16$, Figure $2 \mathrm{~B}$ ). When we compared the frequency of CNA (Figure 2C, 2D), non-clonal ADH cases showed almost no CNA and had an FGA no different to pure ADH $(p=0.60)$, whereas clonal ADH had a significantly higher FGA than pure ADH ( $\mathrm{p}=0.003)$. 
Additionally, for one presumed non-clonal ADH case where no aberrations were identified by LCWGS (S12), we performed high coverage WGS ( 25x) (supplementary material, Supplementary materials and methods) which confirmed the absence of shared somatic mutations or structural aberrations, further supporting an independent origin of the ADH and HG DCIS components.

To summarise the clonal relationships, we performed unsupervised hierarchical clustering analysis (Figure 2E, supplementary material, Figure S8), which showed that 10/13 clonal cases clustered together with their associated carcinoma components. The same ten cases were also called clonal by the R package Clonality (supplementary material, Table S4). Although the three non-clustering cases (S13, S14, S4) shared CNA between ADH and carcinoma components, the latter carried two to three times as many additional CNA as shared events which may explain the lack of apparent clonal relationship. However, they shared multiple breakpoints at a fine resolution, which is highly unlikely to be due to chance and suggestive of true clonal events that might be underestimated by a statistical approach (Figure S6, S7). Both the clustering and Clonality approaches evaluate the balance of CNA, and consider multiple additional events to be indicative of a distant or no relationship. In premalignant breast lesions, shared breakpoints should be emphasized more strongly as suggested previously [26,30], since carcinomas carry a significantly higher burden of CNA, which is not taken into account by Clonality.

\section{Pure ADH lack some CNA frequent in breast carcinoma}


Despite sharing some CNA at similar frequency to carcinomas, other regions of the genome were only rarely observed to be altered in ADH (Table 1). Striking differences were observed for gain of chromosomes $17 \mathrm{q}$ and $21 \mathrm{q}$ as well as loss of chromosome $\mathrm{X}$, none of which were detected in pure ADH but were common in DCIS (Table 1, Figure 1D). Similarly, 11q loss, 11q13.1-11q13.4 high gain, 13q loss, 17p loss and 22q loss were more frequent in DCIS compared with pure ADH (single case each) (Table 1, Figure 1D). Overall, the absence or low frequencies of these CNA in pure ADH but common occurrence in DCIS suggest that these are potential genetic drivers of progression from ADH to DCIS.

Additionally, loss of chromosome 14 or 15 was not observed in ADH nor in LG DCIS, but were frequent events in HG DCIS (14q: 43.2\%; 15q: 32.4\%) and HG IDC (Figure 1D), suggestive of events specific to HG carcinoma. Certain CNA on chromosomes 3, 5, 7, 10 and 18 were also specific to HG carcinoma, although with low frequencies (supplementary material, Table S5). Interestingly, 8q gain and 20q gain were mutually exclusive in pure ADH cases (Figure 1A); however, 26\% cases of HG DCIS showed both 8q gain and 20q gain, indicating that these could be major co-occurring CNA driving HG DCIS. HG DCIS cases lacking $8 \mathrm{q}$ gain (42\%) were more likely to have ERBB2 amplification along with $20 \mathrm{q}$ gain (supplementary material, Figure S9). The remainder carried multiple losses of either 13q, 14q, 15q, 18, 17p along with 20q gain (supplementary material, Figure S9). Thus, to progress from $\mathrm{ADH}$, combinations of $8 \mathrm{q}$ gain and/or 20q gain with other CNA appear to be required for $\mathrm{HG}$ carcinoma development, which is distinct from the LG pathway (e.g. 13q loss was enriched: $1 / 21$ cases for LG DCIS versus $13 / 38$ for HG DCIS, $p=0.02$, Fisher's Exact test). 
Overall, statistically significantly lower CNA frequencies were observed in pure ADH (median FGA 5\%) compared to LG DCIS ( $\mathrm{p}=0.04)$, HG DCIS and IDC (both $\mathrm{p}<0.0001)$ (Figure 1C). Collectively, these data are consistent with ADH being a genetically less advanced lesion compared to carcinomas.

\section{Genetic events associated with malignant progression from ADH}

Clonal ADH harboured specific CNA reflective of those characteristic for the synchronous cancer grade (Table 1), for example, ADH adjacent to HG carcinoma commonly harboured $>2 \mathrm{CN}$ events (6/7) (Figure 1B), including frequent HG-like events such as 8p loss with 8q gain, or 20q gain accompanied by other CNA specific to HG carcinoma such as in chromosomes 13 and 17 (Figure 3). Loss of 11q was significantly more common in clonal $\mathrm{ADH}$ compared to pure $\mathrm{ADH}(5 / 13$ cases versus $1 / 20, \mathrm{p}=0.02)$ and other events were recurrently observed in clonal $\mathrm{ADH}$ but absent from pure $\mathrm{ADH}$ (X loss, 17q gain) or rare in pure $\operatorname{ADH}(13 q$ loss, 22q loss, 17p loss, 11q13.1-q13.4 high gain) (Table 1).

If clonal $\mathrm{ADH}$ represents the less advanced precursor of the synchronous carcinoma, we might expect the carcinoma to contain additional genetic events that promoted progression. Indeed, for 10/13 clonal cases the associated carcinoma had at least one additional CNA. Some of these additional events were seen in multiple cases, for example, 7q gain was seen only in the carcinoma components $(n=4$, e.g. Figure 3 A). Of note, two clonal ADH cases carried CNA absent from the carcinoma suggesting that one subclone of the ADH kept evolving without developing into carcinoma, while another progressed. 
Although most of the synchronous carcinomas were ER+, both the ER- case (S17) (Figure 4) and the ER+/ERBB2 amplified case (S13) (both HG) were clonal with the co-existing ADH. Intriguingly, the ADH component of the latter had gain but not high-level amplification of ERBB2 (Figure 5) with multiple additional gains and amplifications in the HG DCIS across chromosome 17. This profile suggests that low-level gain of ERBB2 may be an early event, but high-level amplification accompanied the progression to carcinoma. In addition, the $\mathrm{CN}$ profiles indicate a highly divergent pattern of evolution whereby the ADH component analysed may have branched off from the clone that evolved into carcinoma at an early phase in the neoplastic expansion, while sharing the same shared breakpoint at chromosome $16 \mathrm{q}$ (supplementary material, Figure S6).

\section{Discussion}

ADH has previously been thought to be a precursor to LG carcinoma only [10-16] based predominantly on the absence of evidence for shared loss of heterozygosity between synchronous ADH with HG cases, and that more than 70\% of LG DCIS and ADH showed 16q loss [1]. However, this conclusion has been based on limited data from low resolution technology. An expression microarray study found that ADH showed a LG gene expression signature and clustered well with LG DCIS/IDC, although with just 7 carcinomas, 5 of which were LG [31]. The exclusion of a precursor relationship between ADH and HG breast cancer 
was mostly driven by lack of data rather than explicit data excluding this possibility. Consequently, this led to one theory that HG carcinoma developed "de novo" [1].

The key limitation of previous studies is their very small sample sizes (average $n=6$ ) [10$16,32-34]$ often due to a large DNA input requirement, which itself leads to a potential sample selection bias, i.e. restricted to larger ADH. A further limitation has been the lack of objective and definitive criteria for diagnosis of $\mathrm{ADH}$ and limited pathological review of the cases. It is likely that some studies have potentially included other types of early neoplasia misdiagnosed as ADH such as FEA or columnar cell hyperplasia, included parts of associated LG DCIS rather than pure lesions or even included residual normal tissue following depletion of ADH cells after diagnostic sectioning $[33,34]$. Consequently, the conclusions drawn by comparing genetic features may be compromised.

In the current study we have overcome previous limitations by combining improved genomic analysis technology with stringent selection and expert pathological review of ADH to explore the molecular landscape and malignant potential of ADH. The high drop-out rate on review of cases previously diagnosed as $\mathrm{ADH}(60 \%$ of cases were reclassified as not $\mathrm{ADH}$, supplementary material, Figure S2) indicates the necessity of our exacting approach.

To our knowledge, our study is the first to demonstrate that a subset of ADH can directly progress to HG carcinoma including ER- or ERBB2 amplified. A clonality study by Larson et al [13] did not clearly state the grades of carcinoma, but it is likely that at least two ADH in their cohort were clonal to HG carcinoma. The genetic events accompanying clonal expansion from $\mathrm{ADH}$ to HG DCIS varied from case to case in our cohort. For example, we 
found that gain of 8q (including the MYC locus) preceded development of carcinoma in two cases, but was a later event in others. Two other synchronous cases showed $20 \mathrm{q}$ gain (including ZNF217 at 20q13.2) instead of 8q gain, along with other $\mathrm{CN}$ events specific to $\mathrm{HG}$ carcinoma (Figure 3C). Data from some previous studies are consistent with our findings, for example 8q gain was reported in two studies of pure $\mathrm{ADH}$; albeit with caveats on sample size $(n=2)[16]$ and data quality [10]. A CGH study of synchronous ADH with IG/HG IDC showed both $8 \mathrm{q}$ gain and 20q gain for 2 out of $3 \mathrm{ADH}$ [32]. It is important to remember that there are inconsistent data available for both pure and synchronous ADH with many limitations in previous studies to consider.

It is well documented that tumours recurring after a diagnosis of DCIS are usually the same grade as the original diagnosis [5,35]; whereas carcinomas diagnosed after an ADH can be of any grade [8]. These studies are in keeping with our data, suggesting that once DCIS has developed it is committed to that molecular pathway, while ADH remains multipotent with respect to progressing to low or high grade, ER+ or ER-, HER2+ or HER2- carcinoma. In other words, our data demonstrates that the model of breast cancer progression with independent "low-grade like" and "high-grade like" pathways could include ADH as a common precursor lesion for any grade. Additionally, our observation that ADH but not LG DCIS can have sub-chromosomal 8q gain may explain why LG DCIS rarely recurs as HG carcinoma, since the latter is characterised by this event ( $>75 \% \mathrm{HG}$ cases shown by others) [1]. The absence of sub-chromosomal 8q gain in LG DCIS was also observed by others [3640]. We speculate that $\mathrm{ADH}$ with such changes could be clinically more significant than LG 
DCIS; the 10-year recurrence rates are similar (10-15\%), but more ADH have the genomic potential to progress to the clinically more aggressive HG carcinoma.

Our cohort had only one ERBB2 amplified DCIS and one ER- IDC case, both of which carried a clonal ADH (itself $E R B B 2$ unamplified/ER+). We found that the $E R B B 2$ amplified DCIS had amplification of ERBB2 unlike the ADH component of this case (Figure 5). One existing FISH study supports our data whereby low-level CN gain was present in the ADH component of the synchronous cases and high-level amplification in the carcinoma [41]. These data support $\mathrm{ADH}$ as a precursor for $\mathrm{ER}+/ E R B B 2$ amplified cases, but as yet it is unknown whether ADH could precede ER-/HER2+ carcinoma, a very aggressive and fastgrowing entity that could overgrow any co-existing ADH. A small subset of tumours occurring after ADH diagnosis were HER2+ (7\%) and ER- (9\%) [8], lower than rates in carcinoma overall [42] yet potentially supporting our finding that $\mathrm{ADH}$ could be clonal to some ER- disease and not only ER+ carcinoma, despite their fundamental differences [1]. This proportion is consistent with the $10 \%$ of IDC with synchronous ADH that were ER[43]. We only had one case of ADH synchronous with ER-/HER2- IDC (clonal), and the genomic profile included $\mathrm{CN}$ characteristics of ER- carcinoma, such as $5 \mathrm{p}$ gain and 10p gain (Figure 4) [29]. Thus, more cases are required to determine the precursor relationship of ADH with ER- carcinoma, as no other study has targeted this group. As ER-/HER2- IDC are associated with BRCA1 mutations, it would be worth investigating if this potential precursor relationship is observed in mutation carriers, patients with family history and/or younger patients. 
A substantial proportion of $\mathrm{ADH}$ were shown to be non-clonal, implying a possible field cancerisation effect [44]. Indeed, Page and Dupont [6] found that cancer developing in the ipsilateral breast after $\mathrm{ADH}$ diagnosis could occur in a different quadrant of the breast and thus possibly non-clonal, unlike most DCIS recurrences [20]. However, due to the unavailability of somatic mutation data, we cannot be completely certain that the non-clonal cases are truly non-clonal, which would lead to an underestimation of the clonality rate.

The increase in CNA, both globally and of particular loci, from ADH to LG and HG carcinoma indicates that these could be later events in breast cancer progression. Indeed, clonal ADH cases were enriched with similar CNA, suggesting they provide a selective advantage for one of the cancer hallmarks [45] either by amplifying regions of oncogenes, such as CCND1 (11q13.1-13.4 gain), or loss of tumour suppressor genes, such as $R B 1$ (13q loss). Although with small sample size, some previous studies also found 11q loss [9,13], or $13 q$ loss $[9,32]$ to be more common in synchronous ADH, and more changes in chromosome 17 in pure DCIS than pure ADH [9]. Moreover, in keeping with previous studies [13,32], most associated carcinoma components showed additional CNA than the synchronous clonal $\mathrm{ADH}$, collectively suggesting that $\mathrm{ADH}$ represents the first clonal expansion of breast cancerized lineages and that additional genetic changes are required to proceed to DCIS and invasive disease of any grade. This model is consistent with other evolutionary studies showing that advanced malignancies tend to have more genetic changes than early tumours [46].

In conclusion, our study is the largest whole-genome study of ADH to date and shows that it has potential to develop into LG and HG breast carcinoma, including ER- and HER2+ 
subtypes. It was previously inexplicable why later development of the tumours after ADH diagnosis were often of $\mathrm{HG} / \mathrm{IG}$ given the prevailing dogma that $\mathrm{ADH}$ could only progress to LG cancer. Our data suggests a model of breast cancer progression (Figure 6) that includes $\mathrm{ADH}$ as a multipotent precursor with different initiating CNA steering progression to either LG or HG carcinoma. This suggests that $\mathrm{CN}$ analysis of $\mathrm{ADH}$ biopsies could be used in the clinical setting to personalize management by identifying which subset of patients are at higher risk of developing cancer, and in particular the clinically more aggressive HG carcinoma. Future studies using ADH cases with extensive follow up are required to compare the molecular profiles of cases that subsequently developed cancer or not. Such a study might enable us to distinguish the cancerized lineages [47] from the non-cancerized lineages whereby the former, in an appropriate micro-environmental context, will drive cancer progression from $\mathrm{ADH}$.

\section{Acknowledgements}

We thank the Peter MacCallum Cancer Centre Bioinformatics and Molecular Genomics core facilities, which were supported by the Australian Cancer Research Foundation. We also thank A/P Ken Opeskin from the Department of Anatomical Pathology, St. Vincent's Hospital for the assessment of lesions and Maria Bisignano from Melbourne Health Pathology Service for coordinating ADH cases. We thank the Nottingham Health Science Biobank and Breast Cancer Now Tissue Bank for the provision of tissue samples.

This study was funded by the Australian National Health and Medical Research Council (APP1063092) and supported by the Peter MacCallum Cancer Foundation. TK was 
supported by a University of Melbourne International Research Scholarship. KLG supported by a Victorian Cancer Agency Mid-Career Fellowship and Union for International Cancer Control Yamagiwa Yoshida Memorial International Study Grant.

\section{Data availability}

Sequence data will be available through NCBI Sequence Read Archive (Bio Project ID: PRJNA522932).

\section{Author contributions statement}

Conception and design: KLG, IGC, GBM, TK; Providing access to clinical samples: GBM, EAR, SBF; Pathological review: PH, AM, JMP, WS; Performing experiments, acquisition of data, analysis and interpretation of data: TK, KLG; Bioinformatics support and data analysis: DLG, MZ, NT, MD; Identification of cases and performing additional experiments: TS, DB, KE, IMM; Drafted the manuscript: TK, KLG, IGC. All authors read and approved the final manuscript; overall study supervision and ethics approval: KLG and IGC.

This article is protected by copyright. All rights reserved. 


\section{References}

1. Lopez-Garcia MA, Geyer FC, Lacroix-Triki M, et al. Breast cancer precursors revisited: molecular features and progression pathways. Histopathology 2010; 57: 171-192.

2. Bombonati A, Sgroi DC. The molecular pathology of breast cancer progression. J Pathol 2011; 223: 307-317.

3. Hartmann LC, Degnim AC, Santen RJ, et al. Atypical hyperplasia of the breast-risk assessment and management options. New Engl J Med 2015; 372: 78-89.

4. Kader T, Hill P, Rakha EA, et al. Atypical ductal hyperplasia: update on diagnosis, management, and molecular landscape. Breast Cancer Res 2018; 20: 39.

5. Lakhani SR, Cancer IAfRo, Organization WH. WHO Classification of Tumours of the Breast. ed). International Agency for Research on Cancer, 2012.

6. Page DL, Dupont WD, Rogers LW, et al. Atypical hyperplastic lesions of the female breast. A long-term follow-up study. Cancer 1985; 55: 2698-2708.

7. Hartmann LC, Radisky DC, Frost MH, et al. Understanding the premalignant potential of atypical hyperplasia through its natural history: a longitudinal cohort study. Cancer Prev Res 2014; 7: 211-217.

8. Visscher DW, Frost MH, Hartmann LC, et al. Clinicopathologic features of breast cancers that develop in women with previous benign breast disease. Cancer 2016; 122: 378-385.

9. O'Connell P, Pekkel V, Fuqua SA, et al. Analysis of loss of heterozygosity in 399 premalignant breast lesions at 15 genetic loci. J Natl Cancer Inst 1998; 90.

10. Gao Y, Niu Y, Wang X, et al. Genetic changes at specific stages of breast cancer progression detected by comparative genomic hybridization. J Mol Med 2009; 87: 145-152.

11. Lakhani S, Collins N, Stratton M, et al. Atypical ductal hyperplasia of the breast: clonal proliferation with loss of heterozygosity on chromosomes 16q and 17p. J Clin Pathol 1995; 48: 611-615.

12. Amari M, Suzuki A, Moriya $\mathrm{T}$, et al. $\mathrm{LOH}$ analyses of premalignant and malignant lesions of human breast: Frequent LOH in $8 p, 16 q$, and $17 q$ in atypical ductal hyperplasia. Oncol Rep 1999; 6: 1277-1280.

13. Larson PS, de las Morenas A, Cerda SR, et al. Quantitative analysis of allele imbalance supports atypical ductal hyperplasia lesions as direct breast cancer precursors. J Pathol 2006; 209: 307-316.

14. Ellsworth RE, Ellsworth DL, Weyandt JD, et al. Chromosomal alterations in pure nonneoplastic breast lesions: implications for breast cancer progression. Ann Surg Oncol 2010; 17: 1688-1694.

15. Gong G, DeVries S, Chew KL, et al. Genetic changes in paired atypical and usual ductal hyperplasia of the breast by comparative genomic hybridization. Clinl Cancer Res 2001; 7: $2410-2414$.

16. Xu S, Wei B, Zhang $\mathrm{H}$, et al. Evidence of chromosomal alterations in pure usual ductal hyperplasia as a breast carcinoma precursor. Oncol Rep 2008; 19: 1469-1476.

17. Nik-Zainal S, Van Loo P, Wedge DC, et al. The life history of 21 breast cancers. Cell 2012; 149: 994-1007.

18. lakovlev VV, Arneson NC, Wong V, et al. Genomic differences between pure ductal carcinoma in situ of the breast and that associated with invasive disease: a calibrated aCGH study. Clin Cancer Res 2008; 14: 4446-4454. 
19. Johnson $\mathrm{CE}$, Gorringe KL, Thompson ER, et al. Identification of copy number alterations associated with the progression of DCIS to invasive ductal carcinoma. Breast Cancer Res Treat 2012; 133: 889-898.

20. Gorringe KL, Hunter SM, Pang JM, et al. Copy number analysis of ductal carcinoma in situ with and without recurrence. Mod Pathol 2015; 28: 1174-1184.

21. Pang J-MB, Savas P, Fellowes AP, et al. Breast ductal carcinoma in situ carry mutational driver events representative of invasive breast cancer. Mod Pathol 2017; 30: 952-963.

22. Kader T, Goode DL, Wong SQ, et al. Copy number analysis by low coverage whole genome sequencing using ultra low-input DNA from formalin-fixed paraffin embedded tumor tissue. Genome Med 2016; 8: 121.

23. Boeva V, Popova T, Bleakley K, et al. Control-FREEC: a tool for assessing copy number and allelic content using next-generation sequencing data. Bioinformatics 2012; 28: 423-425.

24. Scheinin I, Sie D, Bengtsson $\mathrm{H}$, et al. DNA copy number analysis of fresh and formalin-fixed specimens by shallow whole-genome sequencing with identification and exclusion of problematic regions in the genome assembly. Genome Res 2014; 24: 2022-2032.

25. Ostrovnaya I, Begg CB. Testing clonal relatedness of tumors using array comparative genomic hybridization: a statistical challenge. Clin Cancer Res 2010; 16: 1358-1367.

26. Ostrovnaya I, Olshen AB, Seshan VE, et al. A metastasis or a second independent cancer? Evaluating the clonal origin of tumors using array copy number data. Stat Med 2010; 29: 1608-1621.

27. Begg CB, Eng KH, Hummer AJ. Statistical tests for clonality. Biometrics 2007; 63: 522-530.

28. Seshan VE, Olshen AB. DNAcopy: a package for analyzing DNA copy data. 2010; do you mean this 2018 source??

[www.bioconductor.org/packages/devel/bioc/vignettes/DNAcopy/inst/doc/DNAcopy.pdf].

29. Curtis C, Shah SP, Chin SF, et al. The genomic and transcriptomic architecture of 2,000 breast tumours reveals novel subgroups. Nature 2012; 486.

30. Bollet MA, Servant N, Neuvial P, et al. High-resolution mapping of DNA breakpoints to define true recurrences among ipsilateral breast cancers. J Natl Cancer Inst 2008; 100: 48-58.

31. Ma X-J, Salunga R, Tuggle JT, et al. Gene expression profiles of human breast cancer progression. Proc Natl Acad Sci U S A 2003; 100: 5974-5979.

32. Aubele MM, Cummings MC, Mattis AE, et al. Accumulation of chromosomal imbalances from intraductal proliferative lesions to adjacent in situ and invasive ductal breast cancer. Diagn Mol Pathol 2000; 9: 14-19.

33. Weng Z, Spies N, Zhu SX, et al. Cell-lineage heterogeneity and driver mutation recurrence in pre-invasive breast neoplasia. Genome Med 2015; 7: 1.

34. Newburger DE, Kashef-Haghighi D, Weng Z, et al. Genome evolution during progression to breast cancer. Genome Res 2013; 23: 1097-1108.

35. Bijker N, Peterse J, Duchateau L, et al. Histological type and marker expression of the primary tumour compared with its local recurrence after breast-conserving therapy for ductal carcinoma in situ. Br J Cancer2001; 84: 539.

36. Kim SY, Jung S-H, Kim MS, et al. Genomic differences between pure ductal carcinoma in situ and synchronous ductal carcinoma in situ with invasive breast cancer. Oncotarget 2015; 6: 7597. 
37. Vos C, Ter Haar N, Rosenberg C, et al. Genetic alterations on chromosome 16 and 17 are important features of ductal carcinoma in situ of the breast and are associated with histologic type. Br J Cancerr 1999; 81: 1410.

38. Burkhardt L, Grob T, Hermann I, et al. Gene amplification in ductal carcinoma in situ of the breast. Breast Cancer Res Treat 2010; 123: 757-765.

39. James LA, Mitchell EL, Menasce L, et al. Comparative genomic hybridisation of ductal carcinoma in situ of the breast: identification of regions of DNA amplification and deletion in common with invasive breast carcinoma. Oncogene 1997; 14: 1059.

40. Buerger $\mathrm{H}$, Otterbach $\mathrm{F}$, Simon $\mathrm{R}$, et al. Comparative genomic hybridization of ductal carcinoma in situ of the breast-evidence of multiple genetic pathways. J Pathol 1999; 187: 396-402.

41. Xu R, Perle MA, Inghirami G, et al. Amplification of Her-2/neu gene in Her-2/neuoverexpressing and -nonexpressing breast carcinomas and their synchronous benign, premalignant, and metastatic lesions detected by FISH in archival material. Mod Pathol 2002; 15:116-124.

42. Curigliano $G$, Disalvatore $D$, Esposito $A$, et al. Risk of subsequent in situ and invasive breast cancer in human epidermal growth factor receptor 2-positive ductal carcinoma in situ. Ann Oncol 2015; 26: 682-687.

43. Menes TS, Kerlikowske K, Jaffer S, et al. Rates of atypical ductal hyperplasia have declined with less use of postmenopausal hormone treatment: findings from the breast cancer surveillance consortium. Cancer Epidemiol Biomarkers Prev 2009; 18: 2822-2828.

44. Slaughter DP, Southwick HW, Smejkal W. "Field cancerization" in oral stratified squamous epithelium. Clinical implications of multicentric origin. Cancer 1953; 6: 963-968.

45. Hanahan D, Weinberg RA. Hallmarks of cancer: the next generation. Cell 2011; 144: 646674.

46. Nowell PC. The clonal evolution of tumor cell populations. Science 1976; 194: 23-28.

47. Curtius K, Wright NA, Graham TA. An evolutionary perspective on field cancerization. Nat Rev Cancer 2018; 18: 19.

*48. Balleine RL, Webster LR, Davis S, et al. Molecular grading of ductal carcinoma in situ of the breast. Clin Cancer Res 2008; 14: 8244-8252.

*49. Sotiriou C, Wirapati P, Loi S, et al. Gene expression profiling in breast cancer: understanding the molecular basis of histologic grade to improve prognosis. J Natl Cancer Inst 2006; 98: 262-272.

*50. Li H. Aligning sequence reads, clone sequences and assembly contigs with BWA-MEM. arXiv preprint arXiv:13033997 2013.

*51. Cibulskis K, Lawrence MS, Carter SL, et al. Sensitive detection of somatic point mutations in impure and heterogeneous cancer samples. Nat Biotechnol 2013; 31: 213.

*52. Lai Z, Markovets A, Ahdesmaki M, et al. VarDict: a novel and versatile variant caller for nextgeneration sequencing in cancer research. Nucleic Acids Res 2016; 44: e108-e108.

*53. Shen R, Seshan VE. FACETS: allele-specific copy number and clonal heterogeneity analysis tool for high-throughput DNA sequencing. Nucleic Acids Res 2016; 44: e131-e131.

*54. Lee JEA, Li N, Rowley SM, et al. Molecular analysis of PALB2 associated breast cancers. The J Pathol 2018; 245: 53-60. 
*55. Van der Auwera GA, Carneiro MO, Hartl C, et al. From FastQ data to high-confidence variant calls: the genome analysis toolkit best practices pipeline. Curr Protoc Bioinformatics 2013: 11.10. 11-11.10. 33.

*56. Kuilman T, Velds A, Kemper K, et al. CopywriteR: DNA copy number detection from offtarget sequence data. Genome Biol 2015; 16: 49.

*57. Burrell RA, McClelland SE, Endesfelder D, et al. Replication stress links structural and numerical cancer chromosomal instability. Nature 2013; 494: 492-496.

*58. Harvey JM, Clark GM, Osborne CK, et al. Estrogen receptor status by immunohistochemistry is superior to the ligand-binding assay for predicting response to adjuvant endocrine therapy in breast cancer. J Clin Oncol 1999; 17: 1474-1474.

*59. Tuominen VJ, Ruotoistenmäki S, Viitanen A, et al. ImmunoRatio: a publicly available web application for quantitative image analysis of estrogen receptor (ER), progesterone receptor (PR), and Ki-67. Breast Cancer Res 2010; 12: R56.

* Cited only in Supplementary materials

This article is protected by copyright. All rights reserved. 
Table 1. Genetic features in pure ADH, pure LG DCIS, pure HG DCIS and clonal ADH

\begin{tabular}{|c|c|c|c|c|c|}
\hline & \multicolumn{3}{|c|}{ Number of cases $(\%)$} & \multirow{2}{*}{$\begin{array}{c}\text { Number of cases (\%) } \\
\text { Clonal ADH (n=13) grades of } \\
\text { associated carcinoma }\end{array}$} & \multirow{2}{*}{$\begin{array}{c}\text { P value } \\
\text { Pure ADH } \\
\text { versus Clonal } \\
\text { ADH }\end{array}$} \\
\hline $\begin{array}{l}\text { Major and common } \\
\text { CNA of pure ADH }\end{array}$ & $\begin{array}{l}\text { Pure ADH } \\
\qquad(\mathrm{n}=\mathbf{2 0})\end{array}$ & $\begin{array}{l}\text { Pure LG DCIS } \\
\qquad(\mathrm{n}=\mathbf{2 1})\end{array}$ & $\begin{array}{l}\text { Pure HG DCIS } \\
\qquad(\mathrm{n}=\mathbf{3 8})\end{array}$ & & \\
\hline $16 \mathrm{q}$ loss & $13 / 20(65 \%)$ & $17 / 21(80.9 \%)$ & $12 / 38(31.6 \%)$ & 10/13 (76.9\%) 5 HG, 5 LG & 0.7 \\
\hline 1q gain & $5 / 20(25 \%)$ & 10/21 (47.6\%) & $19 / 38(50 \%)$ & 6/13 (46.2\%) $3 \mathrm{HG}, 3 \mathrm{LG}$ & 0.27 \\
\hline 16q loss and 1q gain & $4 / 20(20 \%)$ & $10 / 21(47.6 \%)$ & 9/38 (23.7\%) & 6/13 (46.2\%) 3 HG, 3 LG & 0.14 \\
\hline $\begin{array}{c}\text { Cases with 1q gain also } \\
\text { with } 16 \mathrm{q} \text { loss }\end{array}$ & $4 / 5(80 \%)$ & $10 / 10(100 \%)$ & 9/19 (47.4\%) & $6 / 6(100 \%) 50 \%$ LG, $50 \%$ HG & 0.45 \\
\hline $16 p$ gain & $7 / 20(35 \%)$ & $7 / 21(33.3 \%)$ & 9/38 (23.7\%) & 2/13 (15.4\%) both HG & 0.26 \\
\hline $16 \mathrm{p}$ gain and $16 \mathrm{q}$ loss & $7 / 20(35 \%)$ & $7 / 21(33.3 \%)$ & 6/38 (15.8\%) & 2/13 (15.4\%) both HG & 0.26 \\
\hline 8q gain & $4 / 20(20 \%) ; 3 / 20$ & 0 & $22 / 38(57.9 \%)$ & 4/13 (30.8\%) 3 HG (8q gain), & 0.68 \\
\hline
\end{tabular}

This article is protected by copyright. All rights reserved. 


\begin{tabular}{|c|c|c|c|c|c|}
\hline & $\begin{array}{l}\text { full arm; } 1 / 20 \\
8 \mathrm{q} 22.2-24.22\end{array}$ & & & 1WC 8 gain LG & \\
\hline $8 \mathrm{p}$ loss and $8 \mathrm{q}$ gain & $3 / 20(15 \%)$ & 0 & $14 / 38(36.8)$ & $3 / 13(23.1 \%)$ all $\mathrm{HG}$ & 0.66 \\
\hline 20q gain & $2 / 20(10 \%)$ & $\begin{array}{c}\text { WC } 201 / 21 \\
(4.8 \%) ; 20 \mathrm{q} 13.2(7 \\
\mathrm{Mb}) 1 / 21 \\
\text { Total } 2 / 21(9.5 \%)\end{array}$ & $19 / 38(50 \%)$ & 2/13 (15.4\%) both HG & 1 \\
\hline $20 \mathrm{p}$ loss and 20q gain & $2 / 20(10 \%)$ & 0 & $2 / 38(5.3 \%)$ & 0 & 0.5 \\
\hline 17q loss & $\begin{array}{c}2 / 20(10 \%) \\
(1 / 20: 17 \mathrm{q} 12- \\
25.3) ; 1 / 20: \\
\text { 17q11.2-21.32, } \\
17 \mathrm{q} 24.2-25.3)\end{array}$ & $2 / 21(9.5 \%)$ & $3 / 38(7.9 \%)$ & 1/13 (7.7\%) LG & 1 \\
\hline $\begin{array}{l}\text { CNA associated with } \\
\text { progression to }\end{array}$ & & & & & \\
\hline
\end{tabular}

This article is protected by copyright. All rights reserved. 


\begin{tabular}{|c|c|c|c|c|c|}
\hline carcinoma & & & & & \\
\hline $11 \mathrm{q}$ loss & $1 / 20(5 \%)$ & $5 / 21(23.8 \%)$ & $15 / 38(39.5 \%)$ & $5 / 13(38.5 \%) 2 \mathrm{LG}, 3 \mathrm{HG}$ & 0.02 \\
\hline $13 q$ loss & $1 / 20(5 \%)$ & $1 / 21(4.8 \%)$ & $13 / 38(34.2 \%)$ & $3 / 13(23.1 \%) 2 \mathrm{LG}, 1 \mathrm{HG}$ & 0.28 \\
\hline 13q gain & 0 & 0 & $5 / 38(13.2 \%)$ & $1 / 13(7.7 \%) \mathrm{LG}$ & 0.4 \\
\hline $22 \mathrm{q}$ loss & $1 / 20(5 \%)$ & $4 / 21(19 \%)$ & 8/38 (21.1\%) & $3 / 13(23.1 \%) 2 \mathrm{HG}, 1 \mathrm{LG}$ & 0.27 \\
\hline $\mathrm{X}$ loss & 0 & $4 / 21(19 \%)$ & 8/38 (21.1\%) & 2/13 (15.4\%) both HG & 0.15 \\
\hline $\mathrm{X}$ gain & $\begin{array}{c}1 / 20(5 \%) \\
(X q 11.1-21.1)\end{array}$ & $4 / 21(19 \%)$ & $3 / 38(7.9 \%)$ & $1 / 13(7.7 \%) L G$ & 1 \\
\hline $\begin{array}{c}\text { 11q13.1-q13.4 high } \\
\text { gain }\end{array}$ & $1 / 20(5 \%)$ & $4 / 21(19 \%)$ & $10 / 38(26.3 \%)$ & 2/13(15.4\%) $1 \mathrm{HG}, 1 \mathrm{LG}$ & 0.55 \\
\hline $17 \mathrm{p}$ loss & $1 / 20(5 \%)$ & $3 / 21(14.3 \%)$ & $23 / 38(60.5 \%)$ & 2/13 (15.4\%) both HG & 0.54 \\
\hline
\end{tabular}




\begin{tabular}{|c|c|c|c|c|c|}
\hline 17q gain & 0 & $4 / 21(19 \%)$ & $18 / 38(47.4 \%)$ & $2 / 13(15.4 \%)$ both HG & 0.14 \\
\hline 21q gain & 0 & $1 / 21(4.8 \%)$ & $12 / 38(31.5 \%)$ & 0 & 1 \\
\hline
\end{tabular}

P value from Fisher's Exact test (significant P values are in bold). WC: Whole chromosome; CNA: copy number alterations. All CNA indicate full arm gain/loss unless specified.

This article is protected by copyright. All rights reserved. 
Figure 1. CN analysis of pure ADH and comparison to carcinoma. (A) CNA profile of 20 pure ADH Top: Overall frequency of CNA with chromosome number above. Bottom: Individual sample profiles $(\mathrm{P} 1-\mathrm{P} 20)$. Blue $=$ gain; red $=$ loss. $\mathrm{CNA}<1 \mathrm{Mb}$ have been filtered out to reduce background noise and sequence artefacts. (B) Proportion of cases with different numbers of $\mathrm{CN}$ events observed in pure $\mathrm{ADH}$, DCIS, clonal $\mathrm{ADH}$ with LG cancer, clonal ADH with HG cancer and non-clonal ADH cases. (C) Fraction of the genome altered by CN; mean \pm standard deviation. Unpaired $t$-test with Welch's correction was used for statistical analysis; ${ }^{*} \mathrm{p}<0.05, * * * * \mathrm{p}<0.0001$. D. Frequency plots of $\mathrm{CN}$ gain (blue) and loss (red) with chromosome number on top; comparing pure $\mathrm{ADH}$ with synchronous $\mathrm{ADH}$ and carcinoma (IDC cases from METABRIC). CNA $<1 \mathrm{Mb}$ have been filtered out to reduce background noise and sequence artefacts for ADH and LG DCIS cases.

Figure 2. Clonality of ADH. (A) Number of clonal/non-clonal cases comparing grades of carcinoma. Fisher's Exact test was performed. (B) Number of the clonal/non-clonal cases based on whether they are in the same/different block. (C) Comparison of FGA; mean \pm SD. Mann-Whitney two-tailed test; ** $\mathrm{p}<0.01$, ns; not significant. (D) Overall frequency plots of $\mathrm{CN}$ gain (blue) and loss (red). (E) Unsupervised hierarchical clustering of clonal and nonclonal ADH cases with carcinoma based on CNA segments. Left: the sample ID and the component of the cases $(\mathrm{ADH}=\mathrm{A} / \mathrm{DCIS}=\mathrm{D} / \mathrm{IDC}=\mathrm{I})$ with colour codes; $\mathrm{ADH} / \mathrm{A}=$ pink, $\mathrm{DCIS} / \mathrm{D}=$ green, $\mathrm{IDC} / \mathrm{I}=\mathrm{red}$; the grades of DCIS/IDC: low grade $=1$, intermediate $=2$, high grade $=3$. Row dendrogram coloured by individual sample ID regardless of the components $(\mathrm{ADH} / \mathrm{DCIS} / \mathrm{IDC})$ (i.e. 1 case $=1$ colour). $\mathrm{X}$-axis: Chromosome number on top; bottom: $\mathrm{CN}$ ratio: blue $=$ loss, red=gain. Clonal $\mathrm{ADH}$ cases clustered together $(\mathrm{n}=10)$ marked with black 
asterisks and the remaining 3 clonal ADH cases marked with black hashtag. A larger version of this Figure is available as supplementary material, Figure S8.

Figure 3. Genetic progression of ADH to carcinoma.(A). Typical clonal ADH/HG carcinoma case with gain of chromosome 7 in the DCIS and IDC components and further gain of chromosome 8 in the G3 IDC only. (B) Clonal ADH case with LG carcinoma: CN profiles are identical without additional changes in carcinoma. (C) Clonal ADH case with HG carcinoma where 20q gain along with other CNA preceded the development of carcinoma with additional 18q loss only for carcinoma components. (D) Clonal ADH case with IG carcinoma showing shared 8q gain. (A-D, CN gain in blue and loss in red).

Figure 4. Clonal ADH case with ER negative IDC (S17). The top panel shows two ADH foci which were clonal with ER-ve IDC. ADH are shown with their matched CK5/6 (-ve) and ER immunostaining images(+ve). This $\mathrm{ADH}$ was in a different block and was separated from the tumour site. ER immunostaining is shown for the G3 IDC component (-ve). The bottom panel shows genome-wide copy number profiles: ADH and IDC components shared gain of $5 \mathrm{p}$ and $10 \mathrm{p}$ as well as $\mathrm{Xq}$ loss with additional events for carcinoma components. Red indicates loss and blue indicates gain.

Figure 5. Clonal ADH case with ERBB2 amplified HG DCIS (S13). The top panel shows $\mathrm{H} \& \mathrm{E}$ images for synchronous $\mathrm{ADH}$ foci (ERBB2 unamplified) and $E R B B 2$ amplified $\mathrm{HG}$ DCIS. Genome-wide copy number profile of these two components showed shared events such as $8 \mathrm{q}$ gain, $16 \mathrm{q}$ loss, and $17 \mathrm{p}$ loss, as well as additional events in the DCIS component. 
The bottom panel shows chromosome 17 of both ADH and HG DCIS components. Red indicates loss and blue indicates gain. Red arrow indicates the region of ERBB2.

\section{Figure 6. Revised breast cancer progression model including ADH as a common}

precursor for both LG and HG carcinoma. An unknown event in normal ductal epithelium produces a diploid tumour-initiating cell (46 chromosomes) with a growth advantage that evolves to become $\mathrm{ADH}$. This $\mathrm{ADH}$ might have no CNA and is unable to initiate tumorigenic clonal expansion, remaining as ADH (“evolutionary dead end”). CNA like 16q loss or concomitant 16q loss and 1q gain might be the initiating event for $\mathrm{ADH}$ to progress towards the LG pathway. Once ADH has started along this pathway, limited other CNA such as 11q loss, 11q13.1 high gain and/or 22q loss are the most likely to accompany progression. On the other hand, a subset of $\mathrm{ADH}$ harbouring $8 \mathrm{q}$ gain could directly progress to the HG pathway. $\mathrm{ADH}$ could also harbour or accumulate other HG specific CNA such as $20 \mathrm{q}$ gain, $13 \mathrm{q}$ loss, 17q gain, 17p loss and progress to HG carcinoma. The order of these secondary events may not be crucial. Our model incorporates data from other studies showing that LG and HG IDC have progressed from LG and HG DCIS, respectively. We have focussed on CNA because our data identifies only these events, however, we recognise that individual gene mutations will also be important in this process. 


\section{SUPPLEMENTARY MATERIAL ONLINE}

\section{Supplementary materials and methods Yes}

Supplementary figure legends NO, because legends are embedded with their figures

Figure S1. Representation of pure ADH cases which were re-confirmed or excluded from the initial ADH database.

Figure S2. Patient selection.

Figure S3. Representation of ADH cases synchronous with different grades of carcinoma either re-confirmed or excluded after review.

Figure S4. Examples of shared break points of clonal ADH samples shown in Figure 3C,D.

Figure S5. Examples of shared break points of clonal and non-clonal ADH samples.

Figure S6. Examples of shared break points of two clonal ADH cases that showed discordance using the R Clonality package.

Figure S7. Examples of genome wide copy number profile and shared break points of clonal ADH case (S4) that showed discordance using the R Clonality package.

Figure S8. Correlation plots.

Figure S8. Unsupervised hierarchical clustering of clonal and non-clonal ADH cases with carcinoma based on CNA segments (larger version of Figure 2E)

Figure S9. Correlation plot

This article is protected by copyright. All rights reserved. 
Table S1. Sample information

Table S2. Characteristics of synchronous ADH cases

Table S3. Sequence performance and DNA input

Table S4. Summary of clonal relatedness of ADH cases

Table S5. Other CNA of pure ADH

This article is protected by copyright. All rights reserved. 

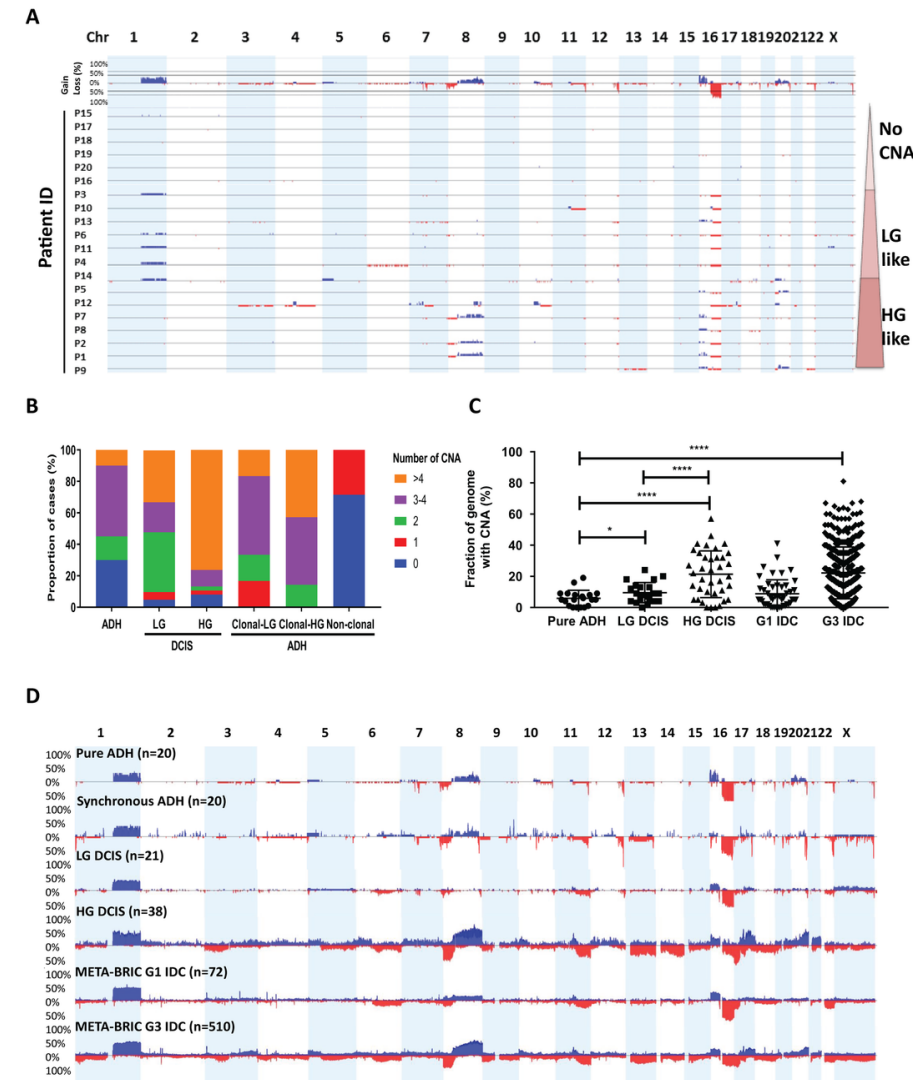

PATH_5262_Fig 1 final.tif 

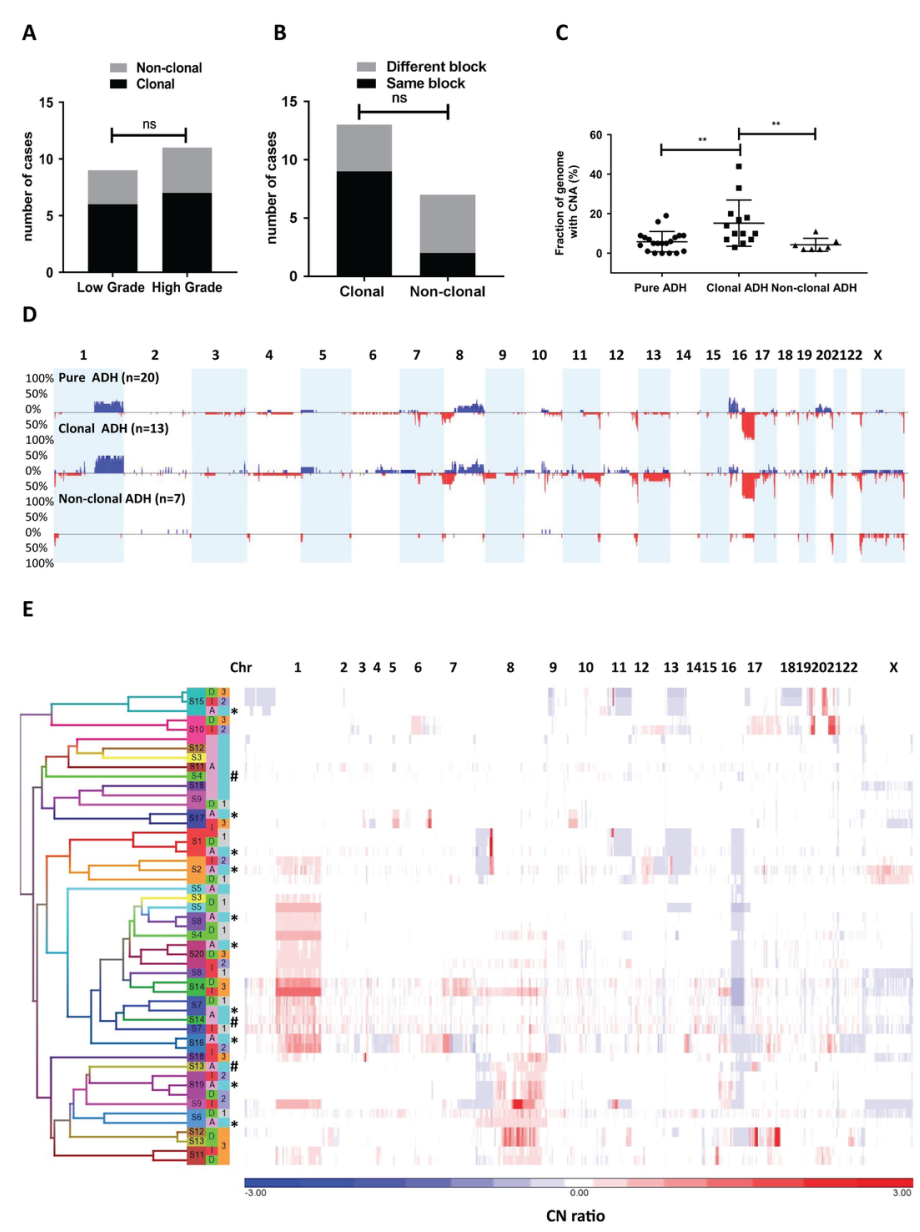

PATH_5262_Fig 2 final.tif

This article is protected by copyright. All rights reserved. 


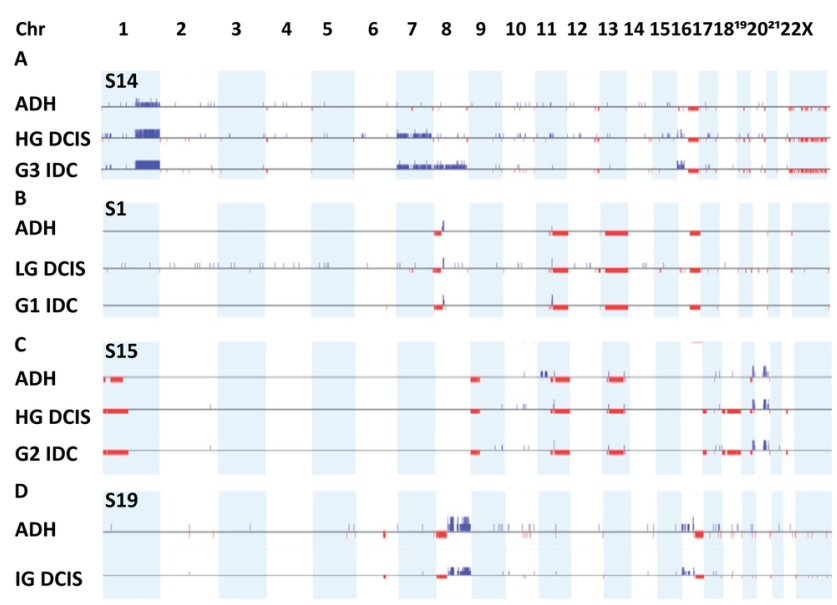

PATH_5262_Fig 3 final.tif

This article is protected by copyright. All rights reserved. 


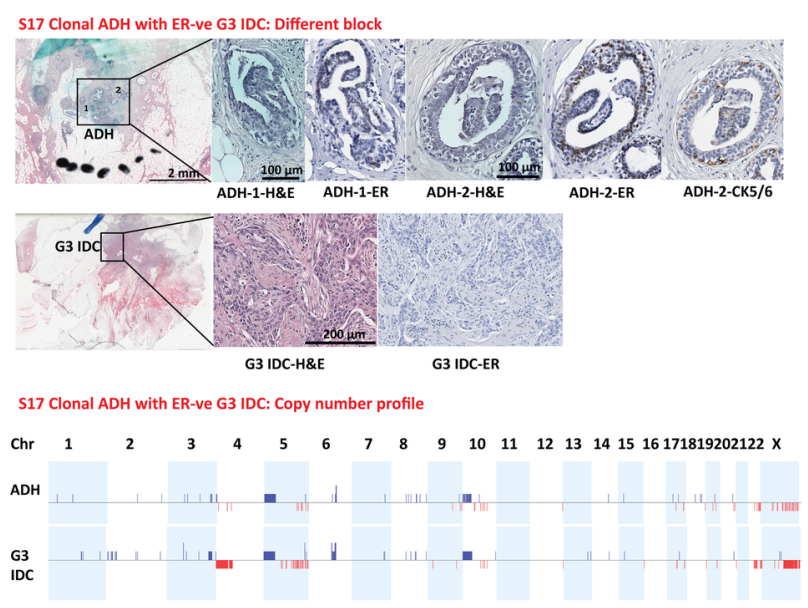

PATH_5262_Fig 4 final.tif

This article is protected by copyright. All rights reserved. 


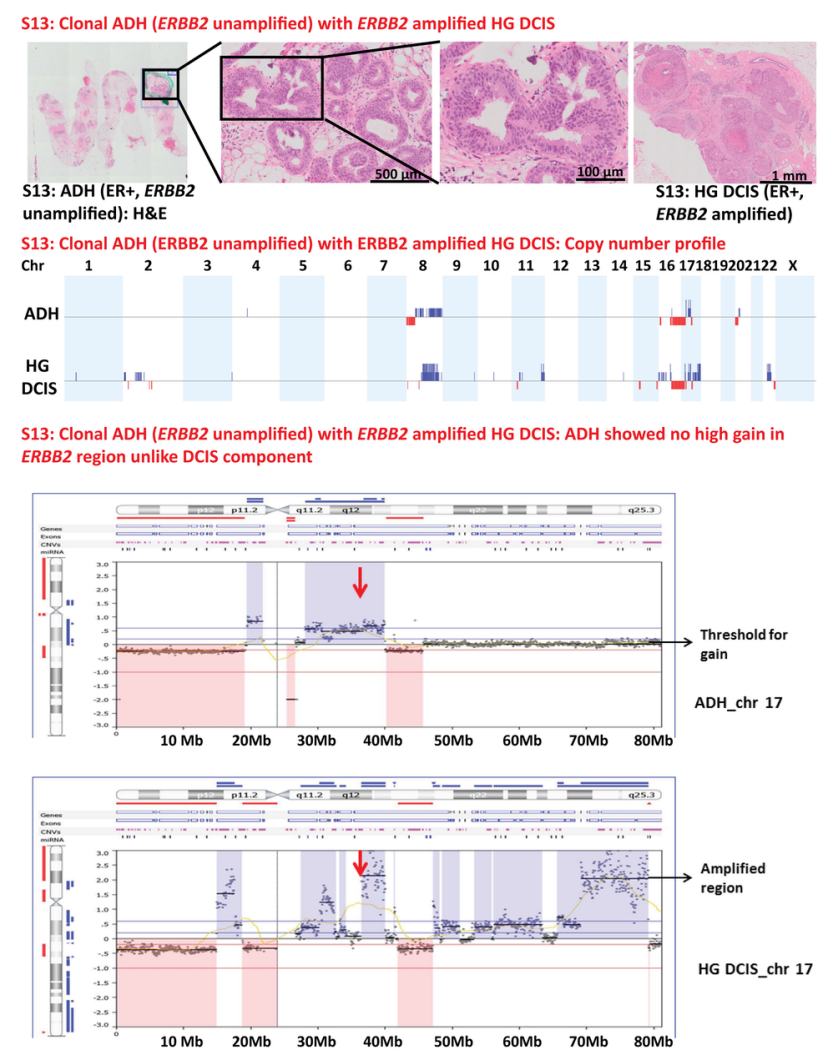

PATH_5262_Fig 5 final.tif

This article is protected by copyright. All rights reserved. 


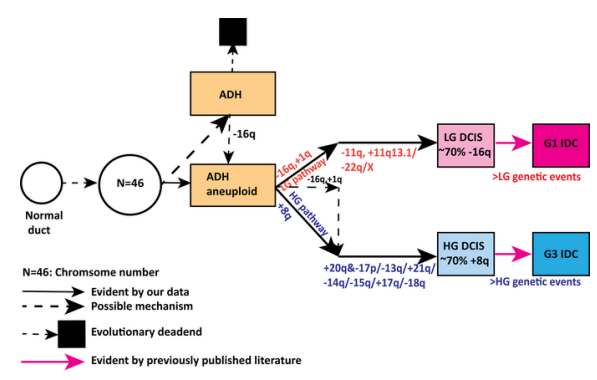

PATH_5262_Fig 6 final.tif

This article is protected by copyright. All rights reserved. 


\section{University Library}

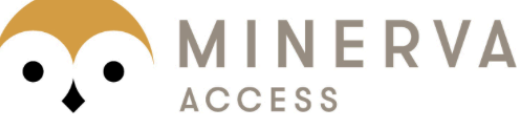

A gateway to Melbourne's research publications

Minerva Access is the Institutional Repository of The University of Melbourne

\section{Author/s:}

Kader, T;Hill, P;Zethoven, M;Goode, DL;Elder, K;Thio, N;Doyle, M;Semple, T;Sufyan, W;Byrne, DJ;Pang, J-MB;Murugasu, A;Miligy, IM;Green, AR;Rakha, EA;Fox, SB;Mann, GB;Campbell, IG;Gorringe, KL

Title:

Atypical ductal hyperplasia is a multipotent precursor of breast carcinoma

Date:

2019-07-01

\section{Citation:}

Kader, T., Hill, P., Zethoven, M., Goode, D. L., Elder, K., Thio, N., Doyle, M., Semple, T., Sufyan, W., Byrne, D. J., Pang, J. -M. B., Murugasu, A., Miligy, I. M., Green, A. R., Rakha, E. A., Fox, S. B., Mann, G. B., Campbell, I. G. \& Gorringe, K. L. (2019). Atypical ductal hyperplasia is a multipotent precursor of breast carcinoma. JOURNAL OF PATHOLOGY, 248 (3), pp.326-338. https://doi.org/10.1002/path.5262.

Persistent Link:

http://hdl.handle.net/11343/285770 